\title{
A INFLUÊNCIA DE REDES INTERPESSOAIS E INTERORGANIZACIONAIS NA INOVAÇÃO DE MPES BRASILEIRAS DE SERVIÇOS
}

\section{THE INFLUENCE OF INTERPERSONAL AND INTERORGANIZATIONAL NETWORKS IN THE INNOVATION OF SERVICE BRAZILIAN SMES}

\author{
Recebido: 12/12/2017 - Aprovado: 14/03/2018 - Publicado: 26/06/2018 \\ Processo de Avaliação: Double Blind Review
}

\author{
Caio Giusti Bianchi ${ }^{1}$ \\ ESPM - Escola Superior de Propaganda e Marketing \\ caio.bianchi@espm.br \\ Welington Rodrigues Alves \\ ESPM - Escola Superior de Propaganda e Marketing \\ w.ralves@hotmail.com

\section{George Rossi} \\ ESPM - Escola Superior de Propaganda e Marketing \\ george@espm.br
}

RESUMO: A inovação é uma importante estratégia de sobrevivência e desenvolvimento para micro e pequenas empresas no Brasil, principalmente por meio de redes multinível como fontes de conhecimento. O objetivo deste estudo é analisar o impacto de redes multinível nos diferentes tipos de inovação de MPEs brasileiras de serviços. Foram realizados mapeamentos cognitivos causais e entrevistas com empreendedores de três empresas, possibilitando a conclusão de que: 1) redes exógenas são mais influentes na inovação de serviços; 2) redes endógenas são mais influentes na inovação de processos; 3) redes com concorrentes são utilizadas principalmente para inovações de marketing; 4) redes com instituições são essenciais para todos os tipos de inovação, capacitação dos empreendedores e redução da mortalidade das MPEs.

Palavras-chave: empreendedorismo; micro e pequenas empresas; inovação; redes multinível.

\footnotetext{
1 Autor para correspondência: ESPM - Escola Superior de Propaganda e Marketing - Rua Dr. Álvaro Alvim, 123 Vila Mariana, São Paulo - SP, 04018-010
}

REMIPE- Revista de Micro e Pequenas Empresas e Empreendedorismo da Fatec Osasco V. 4, No1, jan.-jun. 2018. 
ABSTRACT: Innovation is an important survival and development strategy for micro enterprises and small business in Brazil, mainly through multilevel networks as sources of knowledge. So, the aim of the research is to analyze the impact of multilevel networks on different types of innovation in Brazilian service micro enterprises and small business. Cognitive causal mapping and interviews with entrepreneurs were conducted in three companies, allowing the comprehension that: 1) exogenous networks are more influential in services innovation; 2) endogenous networks are more influential in process innovation; 3) networks with competitors are mainly used for marketing innovation; and 4) networks with institutions are essential for all types of innovation, entrepreneur training and reduction in mortality of micro enterprises and small businesses.

Keywords: entrepreneurship; small enterprises; innovation; multilevel networks.

\section{INTRODUÇÃO}

Empresas de grande porte e multinacionais são atores relevantes para o desenvolvimento econômico de países, porém o papel das micro e pequenas empresas (MPEs) tem sido destaque nos últimos anos. A tipologia de MPEs utilizada no estudo foi criada pelo Serviço Brasileiro de Apoio às Micro e Pequenas Empresas (SEBRAE), um dos principais órgãos de suporte e profissionalização de MPEs. Considera-se micro empresas até nove colaboradores; e pequenas empresas entre 10 e 49 colaboradores (SEBRAE, 2014).

De acordo com a pesquisa do Sebrae (2014, p. 32-36), no universo empresarial brasileiro de mais de nove milhões de MPEs, as empresas foram responsáveis por $27 \%$ do PIB no ano de 2011. Apesar da relevância econômica e social das MPEs, principalmente de comércio e serviço, há uma característica a ser explorada: a alta taxa de mortalidade.

De acordo com Pereira, Grapeggia, Emmendoerfer e Três (2009), a taxa de mortalidade de MPEs, principalmente nos primeiros cinco anos de existência, é maior que a média do mercado. Por conta de tal alta de mortalidade e a relevância econômica, Filion (1999)e Lemos da Silveira Santos, César Alves e Nunes Tavares de Almeida 
(2007) destacaram a baixa capacitação dos empreendedores e as rápidas mudanças no ambiente estratégico como principais fatores de tal fenômeno.

Considerando a necessidade de adaptação e mudanças para sobrevivência de MPEs, a inovação se mostra um fator primordial para que elas mantenham sua relevância econômica (RATTNER, 1984). No contexto de MPEs e da literatura de empreendedorismo, as redes sociais possuem um papel primordial na elaboração e inserção de inovações; sejam elas no nível interpessoal, entre unidades ou entre organizações (TOMAÉL, ALCARÁ e DI CHIARA, 2005).

Por conta da relevância de MPEs e de redes para a sua inovação, o objetivo deste estudo é analisar o impacto de redes interpessoais e interorganizacionais na inovação de MPEs brasileiras de serviços. Para alcançar tal objetivo, a influência de oito tipos de redes em quatro tipos de inovações foi analisada por meio do mapeamento cognitivo causal em oito MPEs brasileiras de serviços. Além do instrumento de mapeamento, entrevistas qualitativas foram realizadas para cruzamento de dados.

\section{REFERENCIAL TEÓRICO}

\subsection{REDES MULTINÍVEL}

O conceito de inovação tem sido abordado na literatura econômica e organizacional há séculos. Conceitos como "inovação aberta" reverberam no âmbito acadêmico atual, porém a relevância de processos interativos de inovação é secular, a exemplo da obra de Schumpeter (1934), publicada inicialmente em 1911. Freeman (1995) reitera a relevância da inovação como um processo interativo, não-linear, endógeno e exógeno de organizações, tendo a interação entre indivíduos, departamentos e ambiente como fatores de inovação.

No contexto da inovação interativa, o conceito de redes emerge pela sua capacidade de tipificar e conceituar os diferentes níveis e tipos de interação supracitados. Considerando que o campo de redes possui aplicação em diversas áreas do conhecimento, a fim de manter tal característica, Brass, Galaskiewicz, Greve e Tsai (2004, p. 795) definem redes como "um conjunto de nós e ligações representando algum relacionamento entre tais nós; sendo eles agentes individuais, unidades de trabalho ou organizações"(p. 795). 
Apesar de pesquisas de redes terem o potencial de aplicação em diversas áreas desde pessoas até componentes eletrônicos -, no âmbito de estudos organizacionais, a abordagem multinível de Brass et al. (2004), a partir de revisões da literatura, se faz relevante; sendo classificada em: redes entre pessoas, redes entre unidades e redes entre organizações. A aplicação da tipologia de redes apresentada por Brass et al. (2004) pode ser observada em diversos contextos, como observado em Provan e Kenis (2008), Borgatti, Mehra, Brass e Labianca (2009), Gawer e Cusumano (2014) e Rank (2014).

O nível micro da análise de redes conta com indivíduos e suas relações interpessoais como ligações. Nesse caso, cada indivíduo conta com características, necessidades e reações específicas, formando uma rede baseada em relações formais e informais (TOMAÉL ET AL., 2005). As relações formais são influenciadas por questões hierárquicas e de cultura organizacional, ilustradas por interações entre indivíduos em ambientes formais e pré-determinados, como reuniões e negociações. Já as relações informais acontecem entre indivíduos em ambientes informais e imprevistos, como pausas para o café ou encontros após o expediente (KILDUFF e TSAI, 2003).

O nível meso da análise de redes diz respeito às unidades organizacionais, consideradas os nós da rede, enquanto as interações formadas entre si são consideradas as ligações. A definição de "unidades" varia por conta da estrutura organizacional de cada empresa, porém é possível distinguir a interação com unidades internas e com unidades externas (BRASS ET AL., 2004). Apesar de o tipo de interação entre unidades ser semelhante, como o desenvolvimento de um novo produto, a proximidade física e a intensidade das relações sociais informais diferenciam a interação entre unidades internas, como departamentos de uma mesma unidade; e unidades externas, como filiais e subsidiárias (EMIRBAYER e GOODWIN, 1994).

O nível macro de análise de redes aborda a interação com organizações (empresariais, instituições ou clientes) como nós, e acordos, parcerias e cooperação como ligações. Com uma abordagem próxima de estudos organizacionais tradicionais, as redes organizacionais são formadas a partir de empresas detentoras de recursos que possuem interesse em tomar decisões conjuntas para o seu uso(DE PELLEGRIN, BALESTRO, JUNIOR e CAULLIRAUX, 2007). Considerando a cadeia de valor como base, há quatro direções pelas quais tais redes podem se desenvolver: fornecedores, clientes, concorrentes e instituições (como universidades, institutos de pesquisa e órgãos de apoio à inovação)(BRASS ET AL., 2004). 
Com isso, o Quadro 1 sumariza os oito tipos de redes que representam os conceitos a serem analisados no presente estudo.

Quadro 1. Conceitos de Redes

\begin{tabular}{|c|c|c|}
\hline Conceito & Definição & Referências \\
\hline $\begin{array}{l}\text { Interpessoais } \\
\text { Formais }\end{array}$ & $\begin{array}{l}\text { Rede formada pelas relações entre indivíduos de uma mesma } \\
\text { empresa, regidas pela hierarquia e limitadas ao ambiente } \\
\text { profissional. }\end{array}$ & \multirow{2}{*}{$\begin{array}{lr}\text { (TOMAÉL } & \text { ET } \\
\text { AL., } 2005) ; \\
\text { (KILDUFF } \\
\text { TSAI, 2003) }\end{array}$} \\
\hline $\begin{array}{l}\text { Interpessoais } \\
\text { Informais }\end{array}$ & $\begin{array}{l}\text { Rede formada pelas relações entre indivíduos de uma mesma } \\
\text { empresa, regidas por características em comum e afinidades } \\
\text { pessoais. }\end{array}$ & \\
\hline $\begin{array}{l}\text { Entre Unidades } \\
\text { Internas }\end{array}$ & $\begin{array}{l}\text { Rede formada pelas relações formais entre departamentos, } \\
\text { unidades de negócios e grupos da mesma empresa. }\end{array}$ & \multirow{2}{*}{$\begin{array}{l}\text { (BRASS ET AL., } \\
\text { 2004); } \\
\text { (EMIRBAYER e } \\
\text { GOODWIN, } \\
\text { 1994) }\end{array}$} \\
\hline $\begin{array}{l}\text { Com Unidades } \\
\text { Externas }\end{array}$ & $\begin{array}{l}\text { Rede formada pelas relações formais entre filiais ou } \\
\text { subsidiárias da mesma empresa. }\end{array}$ & \\
\hline $\begin{array}{c}\text { Com } \\
\text { Fornecedores }\end{array}$ & $\begin{array}{l}\text { Rede formada pelas relações entre empresa e fornecedores de } \\
\text { produtos ou serviços (a montante da cadeia de valor). }\end{array}$ & \multirow{4}{*}{$\begin{array}{l}\text { (DE PELLEGRIN } \\
\text { ET AL., 2007); } \\
\text { (BRASS ET AL., } \\
\text { 2004) }\end{array}$} \\
\hline Com Clientes & $\begin{array}{l}\text { Rede formada pelas relações entre empresa e clientes (a jusante } \\
\text { da cadeia de valor). }\end{array}$ & \\
\hline $\begin{array}{c}\text { Com } \\
\text { Concorrentes } \\
\end{array}$ & $\begin{array}{l}\text { Rede formada pelas relações entre empresa e concorrentes } \\
\text { diretos e indiretos no mercado. }\end{array}$ & \\
\hline Com Instituições & $\begin{array}{l}\text { Rede formada pelas relações entre empresa e instituições } \\
\text { externas, como institutos de pesquisa, de suporte ao } \\
\text { desenvolvimento e universidades. }\end{array}$ & \\
\hline
\end{tabular}

Elaborado pelos autores.

\subsection{TIPOLOGIA DE INOVAÇÃ̃O}

A inovação tem sido utilizada para garantir a competitividade das empresas no mercado, visto que este tem estado constantemente em mudança. Ela pode ser definida como um conjunto de ações estratégicas de caráter evolutivo que transformam os métodos de produção e ambiente organizacional, sendo capaz de criar novos produtos e mercados (SCHUMPETER, 1988). Porter (1986)define inovação como o conjunto de melhorias que são efetuadas na tecnologia ou maneira de fazer as coisas.

Há diversas ramificações e especificações da tipologia de inovações de acordo com objetivos específicos. O Manual da PINTEC (Pesquisa de Inovação) do IBGE (2015), principal meio de mensuração da inovação corporativa brasileira, limita inovações às classificações de produto e processo. Já o Manual de Oslo (OCDE, 2005), elaborado pela Organização para Cooperação e Desenvolvimento Econômico, é referência em tal tipologia por conta da sua abrangência e montante de aplicações 
empíricas. Sua tipologia contempla inovações de produto/serviço, de processo, organizacional e de marketing.

A inovação de produto consiste na introdução de um produto ou serviço novo ou aprimorado, levando em consideração suas características físicas e utilização(OCDE, 2005). Complementando, a PINTEC(IBGE, 2015), considera que um produto novo é aquele cujas principais caraterísticas diferem de todos os produtos existentes no atual portfólio da empresa e que o produto melhorado ou aprimorados e refere a um produto já existente cujo desempenho foi aperfeiçoado por meio da modificação ou incremento estruturais (MEDEIROS, RIBEIRO e CORTIMIGLIA, 2014).

Ainda, quando se trata de serviços, a inovação pode estar presente em novos conceitos, ideias ou soluções para um problema, sendo capaz de reduzir custos e agregar valor. Em relação à melhoria do serviço, pode estar relacionada com mudanças na forma como é oferecida ao cliente, tendo como objetivo aumento da eficiência e agilidade na sua prestação (IBGE, 2015; LUSCH e NAMBISAN, 2015).

A inovação de processo está relacionada à implantação de uma metodologia de produção ou logística nova ou significativamente melhorada, incluindo-se técnicas, softwares ou equipamentos(OCDE, 2005). Acrescentando, de acordo com a PINTEC (IBGE, 2015), a inovação de processo deve apresentar aumento significativo da qualidade dos produtos/serviços ou redução dos custos de produção e entrega(UN e ASAKAWA, 2015).

No entanto, Colossi (2004)ressalta que a inovação não deve ser atrelada somente aos âmbitos de produto e processo, visto que as relações pessoais e as formas de gestão, assim como a relação da empresa com mercado, também fazem parte das estratégias organizacionais e merecem o devido destaque.

Quanto à inovação de marketing, são consideradas implantações de novos métodos de marketing antes não utilizados pela empresa, que vão desde alterações significativas na concepção ou embalagem do produto até a fixação de preços, promoções e posicionamento no mercado. Essa inovação está voltada para atender as necessidades identificadas dos clientes e introdução da empresa em novos mercados com intuito de aumentar as vendas (OCDE, 2005).

De acordo com Kotler e Keller (2006) e Gupta, Malhotra, Czinkota e Foroudi (2016), as estratégias de marketing devem estar em sintonia com o mercado para que as necessidades e desejos dos clientes sejam identificados. Com isso, as inovações de 
marketing passam a ter condições necessárias para que ações sejam desenvolvidas visando aumentar a competitividade empresarial.

Neste sentido, segundo o Manual de Oslo (OCDE, 2005), a inovação organizacional consiste na implementação de novos métodos de gestão organizacional, modificações no ambiente de trabalho ou em suas relações externas. Os principais objetivos da implantação de uma inovação organizacional são redução de custos administrativos e de suprimentos e promoção da satisfação dos colaboradores no ambiente laboral. Ainda, segundo Aranda (2009) e Camisón e Villar-López (2014), a inovação organizacional pode ser utilizada para garantir vantagem competitiva por meio da criação, desenvolvimento e implementação de novos métodos de gestão capazes de otimizar os recursos estratégicos empresariais.

Ademais, um aspecto a ser considerado, principalmente na dinamicidade do mercado contemporâneo, é a possível implantação conjunta de diferentes tipos de inovações. Isso significa que uma ação específica é capaz de abarcar mais de um tipo de inovação na empresa, como a melhoria na embalagem de um produto (inovação de marketing) levando à necessidade de uma nova forma de distribuição (inovação de processo), a exemplo da revisão de literatura de Greenhalgh, Robert, Macfarlane, Bate e Kyriakidou (2004).

A tipologia de inovação utilizada na pesquisa pode ser observada no Quadro 2. Os quatro tipos de inovação, suas definições e respectivas referências embasam a pesquisa empírica apresentada. 
Quadro 2. Conceitos de Inovação:

\begin{tabular}{|c|l|l|}
\hline Conceito & \multicolumn{1}{|c|}{ Definição } & \multicolumn{1}{|c|}{ Referência } \\
\hline \multirow{3}{*}{$\begin{array}{c}\text { Produtos e } \\
\text { Serviços }\end{array}$} & $\begin{array}{l}\text { Introdução de um produto ou serviço novo ou melhorado nas } \\
\text { características ou usos. Incluem-se melhoramentos } \\
\text { significativos em características funcionais, como } \\
\text { especificações técnicas, softwares incorporados e facilidade } \\
\text { de uso. }\end{array}$ & $\begin{array}{l}\text { (OCDE, 2005); (IBGE, } \\
\text { ET AL., 2014); (LUSCH } \\
\text { e NAMBISAN, 2015) }\end{array}$ \\
\hline \multirow{5}{*}{ Processo } & $\begin{array}{l}\text { Implementação de um método de produção ou distribuição } \\
\text { novo ou significativamente melhorado. Incluem-se } \\
\text { mudanças significativas em técnicas, equipamentos e/ou } \\
\text { softwares. }\end{array}$ & $\begin{array}{l}\text { (OCDE, 2005); (IBGE, } \\
\text { ASAKAWA, 2015) }\end{array}$ \\
\hline $\begin{array}{l}\text { Implementação de um novo método de marketing com } \\
\text { mudanças significativas na concepção ou posicionamento } \\
\text { do serviço, em sua promoção ou na fixação de preços. }\end{array}$ & $\begin{array}{l}\text { (OCDE, } \\
\text { (KOTLER e KELLER, } \\
\text { 2006); (GUPTA ET AL., } \\
\text { 2016) }\end{array}$ \\
\hline Organização & $\begin{array}{l}\text { Implementação de um novo método organizacional nas } \\
\text { práticas de negócios da empresa, na organização do seu } \\
\text { local de trabalho ou em suas relações externas. }\end{array}$ & $\begin{array}{l}\text { (OCDE, } \\
\text { (ARANDA, } \\
\text { (CAMISÓN e VILLAR- } \\
\text { LÓfEZ, 2014) }\end{array}$ \\
\hline
\end{tabular}

Elaborado pelos autores.

\section{METODOLOGIA DE PESQUISA;}

A pesquisa possui uma abordagem qualitativa e exploratória para a compreensão do fenômeno em questão(COOPER e SCHINDLER, 2016). Os aspectos qualitativos e exploratórios são relevantes pois os pesquisadores buscam compreender de que maneira diferentes níveis de redes influenciam na inovação de MPEs brasileiras de serviços, a partir do ponto de vista dos proprietários das empresas. O método de pesquisa utilizado para a compreensão do fenômeno é o estudo de caso múltiplo, permitindo que os pesquisadores identifiquem comunalidades e diferenças entre os casos analisados (YIN, 2013).

Para a coleta e análise de dados, a técnica de mapeamento cognitivo causal foi aplicada. De acordo com Eden (1988), ela é útil para representar como indivíduos compreendem um fenômeno cognitivamente. Logo, mapas cognitivos causais permitem a compreensão da percepção dos entrevistados sobre a interação entre dois conceitos representados na pesquisa por Redes e Tipos de Inovação (SCAVARDA, BOUZDINCHAMEEVA, GOLDSTEIN, HAYS e HILL, 2004).

De acordo com o Serviço Brasileiro de Apoio às Micro e Pequenas Empresas(SEBRAE, 2014), as MPEs compõem 99\% do volume total de empresas brasileiras, empregando mais de $50 \%$ da mão de obra nacional gerando $27 \%$ do Produto Interno Bruto do país. Então, a amostra da pesquisa é composta por micro e pequenas 
empresas brasileiras de serviços por conta da sua protagonização no desenvolvimento econômico brasileiro.

Para potencializar os resultados obtidos pela pesquisa, compuseram a amostra somente empresas que estão em processo de inovação e, para aplicar tal critério, empresas participantes do programa ALI (Agentes Locais de Inovação) do Sebrae-SP foram selecionadas. O projeto do Sebrae oferece acompanhamento na implantação de inovações em micro e pequenas empresas em diversas cidades do Brasil.

Como o projeto em questão abrange diversos setores da economia brasileira, foram selecionadas oito empresas que estão em processo de inovação e compõem diferentes setores de serviços, sendo seus proprietários os entrevistados. A diversidade de setores se faz relevante para que os resultados da pesquisa não sejam limitados a uma área específica, potencializando a reprodutibilidade dos mesmos (LAPERRIÈRE, 2008).

Dessa maneira, a amostra final de pesquisa é composta por empresas dos setores de: cursos profissionalizantes na área de beleza (E1), varejo de produtos naturais (E2), serviços de automação residencial (E3), educação infantil (E4), varejo de móveis (E5), serviços de beleza (E6), alimentação (E7) e varejo de alimentos orientais (E8).

\section{APRESENTAÇÃO DE RESULTADOS E DISCUSSÃO;}

A aplicação do instrumento de coleta de dados e as entrevistas permitem que os pesquisadores observem quais redes possuem influência em quais tipos de inovações nas MPEs brasileiras de serviços. Por terem sido realizadas oito entrevistas, análises estatísticas de distância entre mapas e significância não são possíveis, sendo os resultados apresentados em uma matriz de influência e em um mapa causal cognitivo com base nos dados coletados.

A matriz com a soma das respostas dos entrevistados é apresentada na Tabela 1. Nela, é possível observar quais tipos de redes possuem maior influência nas inovações, considerando que os valores são a soma das respostas $(\mathrm{de}-3 \mathrm{a}+3)$ dos oito entrevistados. Apesar de o instrumento contar com a possibilidade de influência negativa ou positiva, nenhum entrevistado avaliou influências negativas - levando à tabela com apenas valores positivos. 
Tabela 1 - Matriz Acumulada de Influência entre Variáveis:

\begin{tabular}{c|cccccccc}
$\begin{array}{c}\text { Redes/ } \\
\text { Inovação }\end{array}$ & $\begin{array}{c}\text { Interp. } \\
\text { Formai } \\
\text { s }\end{array}$ & $\begin{array}{c}\text { Interp. } \\
\text { Informai } \\
\text { s }\end{array}$ & $\begin{array}{c}\text { Unidades } \\
\text { Internas }\end{array}$ & $\begin{array}{c}\text { Unidades } \\
\text { Externas }\end{array}$ & $\begin{array}{c}\text { Fornece- } \\
\text { dores }\end{array}$ & Clientes & $\begin{array}{c}\text { Concor- } \\
\text { rentes }\end{array}$ & $\begin{array}{c}\text { Institui- } \\
\text { ções }\end{array}$ \\
\hline $\begin{array}{c}\text { Produtos/S } \\
\text { erviços }\end{array}$ & 14 & 1 & 12 & 3 & 19 & 19 & 16 & 19 \\
Processo & 17 & 5 & 14 & 3 & 8 & 14 & 6 & 20 \\
Marketing & 13 & 1 & 9 & 3 & 16 & 3 & 20 & 23 \\
$\begin{array}{c}\text { Organi- } \\
\text { zação }\end{array}$ & 7 & 2 & 9 & 0 & 7 & 3 & 1 & 19 \\
\hline
\end{tabular}

Um aspecto a ser considerado na Tabela 1 é o valor da influência da rede com unidades externas. Considerando que unidades externas são definidas como filiais, franquias ou subsidiárias da empresa focal, apenas um dos oito entrevistados era parte de uma franquia. Observa-se que o valor apresentado na coluna de Unidades Externas representa apenas o E8, enquanto as demais colunas representam a soma das respostas dos oito entrevistados.

Analisando a influência das redes nas inovações, as redes interpessoais formais possuem a maior influência nas inovações de processo. Segundo os entrevistados, a interação formal entre os colaboradores das empresas permite a troca de experiências e expectativas em relação ao cotidiano, permitindo a emergência de inovações de processo.

O principal aspecto a ser considerado nas redes interpessoais formais é o impacto de reuniões periódicas para feedback da equipe sobre as condições de trabalho e sugestões de melhoria. Tal impacto é exemplificado por excertos das entrevistas:

Temos uma reunião para revisão de processos e temos muita ajuda nesse sentido por parte do corpo técnico e administrativo. (E3).

Professoras são muito participativas e impactam muito nos processos, principalmente no que diz respeito à articulação com a secretaria e departamento responsável por materiais, impressões etc. (E4).

Os funcionários sempre apontam os problemas nas reuniões e sempre alinhamos as soluções e o que é melhor para todos e desempenho da equipe." (E7).

Os funcionários contribuem bastante para os processos, se não está muito bom, eles falam e chegamos a um acordo. (E8).

Em compensação, as redes interpessoais informais são as redes de menor impacto na inovação em todas as empresas da amostra. De acordo com as entrevistas, apesar de 
haver momentos de descontração e interação social fora do ambiente de trabalho, aspectos referentes a inovações e melhorias são concentrados no ambiente formal.

A inovação de processo é a mais influenciada pelas redes interpessoais formais, sendo que, sua pontuação baixa é representada pela troca de conhecimento entre os colaboradores em processos que são realizados no ambiente de trabalho ou oportunidades ambientais de encontros informais.

Saem poucas ideias, uma vez por exemplo, melhoramos uma propaganda que um bar em que fomos estava fazendo e implantamos na empresa. (E3).

Os funcionários não tratam muito desses assuntos de trabalho fora do salão. Às vezes comentam alguma técnica e trocam informações entre si, mas nada em relação à melhoria para o salão como um todo. (E6).

A rede entre unidades internas, por outro lado, possui influência média em todos os tipos de inovação. Entretanto, um aspecto destacado nas entrevistas é o fato de a troca de conhecimento entre unidades internas ser um processo contínuo e de longo prazo. Logo, a influência da rede entre unidades internas se mostra como uma fonte contínua e estável de inovação, ao contrário da influência pontual dos clientes e fornecedores, por exemplo.

\footnotetext{
A cozinha fala muito com o atendimento e vice-versa, inclusive implantamos plaquinhas de espera do suco para que o preparo fosse agilizado. Antes o suco às vezes não chegava ao cliente por falta de comunicação ou chegava depois que o cliente tinha terminado sua refeição. (E7).

A cozinha está sempre em conexão com o pessoal de vendas e sempre que há algo que os atendentes repassam à cozinha, até mesmo quanto à apresentação do prato e tudo mais. (E8).
}

A influência das redes com unidades externas é um ponto a ser destacado nos resultados. Apesar de apenas um dos oito entrevistados na pesquisa ser parte de uma franquia e, consequentemente, ser a única empresa com uma possível rede com unidades externas, os resultados foram enfáticos.

De acordo com o entrevistado 8, uma unidade com maior experiência e influência é fonte de diversos conhecimentos para o entrevistado, desde a inovação de produtos até o marketing. Porém, o destaque está para a ausência de influência da rede com unidades externas na inovação organizacional. O entrevistado afirma que, apesar de a rede cooperar com algumas inovações, a empresa possui um modo próprio de gestão que não é influenciado externamente: "A unidade de Indaiatuba sempre nos repassa os 
fornecedores, novidades de produtos e pratos, e tentamos sempre adequar à nossa realidade". (E8).

Redes com fornecedores possuem uma das maiores influências nas inovações. Dos quatro tipos de inovação analisados, a rede possui maior impacto em inovações de produtos e serviços e em inovações de marketing. O impacto ocorre porque tanto produtos e serviços como marketing são os principais pontos de contato com a empresa focal. Em compensação, o impacto em inovações internas à empresa, como de processo e organizacional, é reduzido.

A rede com fornecedores possibilita, principalmente, que os mesmos apresentem aos entrevistados novos produtos e serviços no mercado. Com isso, os insumos necessários para que as empresas focais possam inovar são inseridos. Fornecedores também são fontes de novas estratégias de marketing atreladas a tais insumos, com forte impacto nas inovações de marketing.

O fornecedor do sistema de ensino fornece pelo formato EAD algumas capacitações para as professoras e isso auxilia muito em termos de inovação e novas metodologias. (E4).

Os fornecedores trazem tudo que é de novo e está "pipocando" no mercado. (E5).

Trazem muitos folders e banners para que possamos vender seus produtos ou usá-los nos procedimentos, isso impacta bastante (E6).

Os fornecedores sempre nos ajudam com algumas ações de marketing, como promoções e datas comemorativas japonesas. (E8).

As redes com clientes também possuem impacto relevante em diferentes tipos de inovação. Apesar de possuírem baixo impacto em inovações de aspectos burocráticos das empresas da amostra, como inovações de marketing e organizacionais, possuem alto impacto nos dois pontos que estão em contato: as inovações de produtos/serviços e de processo.

Assim como nas redes com fornecedores, as redes com clientes influenciam os pontos de contato com as empresas focais. No caso da amostra de pesquisa, clientes possuem papel ativo em sugerir mudanças e melhorias nos produtos e serviços oferecidos, assim como no processo que os envolvem.

Eles ajudam muito, sempre procuram novidades, às vezes até antes de lançarmos. Por exemplo, deram a dica de e-commerce e, também, delivery. (E2).

Os clientes sempre trazem novidades e sugestões de procedimentos, querem sempre o esmalte, penteado dos artistas do momento e com isso vem os novos 
serviços. Temos que estar sempre ligados no que está acontecendo no momento. (E6).

Os clientes sempre contribuem com o cardápio e o adequamos quando está ao nosso alcance, além de sugestões voltadas ao atendimento, como o suco com atraso e numeração das mesas que não tínhamos. (E7).

As redes com concorrentes, também, são uma das principais fontes de inovação dos entrevistados. A rede possui grande impacto em inovações de produtos/serviços e de marketing, conhecimentos que são facilmente acessíveis e pouco sensíveis, em relação aos aspectos processuais e organizacionais.

A influência dessas redes se dá, principalmente, pelo alinhamento entre as empresas sobre o que é mais eficiente na captação e satisfação dos consumidores. Com isso, tanto as empresas focais como seus concorrentes se aproximam no desempenho de produtos/serviços ofertados e nas estratégias de divulgação.

Um ponto a ser destacado na rede com concorrentes é que, apesar de haver interação e troca de conhecimento entre as empresas focais e seus concorrentes, a abordagem da interação é essencialmente competitiva. Não foram identificados, na amostra de pesquisa, aspectos de cocriação ou parceria para além de divulgações pontuais.

Sempre conversamos sobre como e quais os produtos nossos concorrentes estão trabalhando, então a rede impacta bastante. O mercado dita muitas coisas. (E3).

Sempre acompanhamos as campanhas dos concorrentes e às vezes fazemos algumas parecidas ou melhoramos as que eles estão efetuando naquele momento. (E5).

Ficamos de olho no Facebook dos concorrentes e nos movimentamos conforme eles também se movimentam, uma vez fizemos um sorteio de curtir e compartilhar um post que o concorrente fez. (E6).

Redes com instituições são o último tipo de rede a ser apresentado. Apesar de o impacto das redes com instituições ser esperado por conta da amostra de pesquisa participar do programa ALI do Sebrae-SP, as entrevistas possibilitaram maior compreensão da relevância do tipo de rede. Os entrevistados também participam de outras redes institucionais de apoio à inovação, como a interação com parques tecnológicos e SENAI. 
O principal ponto a ser considerado, além de haver alto impacto em inovações de produto/serviço, processo e marketing, é a rede com instituições como a única rede que possui alto impacto na inovação organizacional.

\footnotetext{
Inovamos mais com o Sebrae mesmo, com ajuda do ALI. As universidades não ajudam, mas deveriam ajudar mais. (E2).

O parque tecnológico ajuda bastante. SENAI também. E o Sebrae com a consultoria (diversificamos os produtos). (E3).

O programa ALI ajuda com alguns serviços novos que estamos oferecendo, como dia do noivo, porque antes tínhamos somente o dia da noiva. (E6).

SEBRAE e ALI, participamos do PAS (Programa Alimento Seguro) e fizemos diversas alterações na cozinha em relação aos processos e manipulação dos alimentos. (E8).
}

A análise da influência de cada rede nas inovações possibilita a compreensão dos principias pontos a serem considerados em cada rede para empresas alcançarem diferentes tipos de inovações. Porém, com foco nos quatro tipos de inovação abordados, é possível identificar padrões nos dados coletados, que possibilitam uma análise das suas principais características.

As inovações de produtos e serviços são as mais influenciadas pelas redes em geral. Por serem micro e pequenas empresas que buscam a diferenciação para alcançar destaque no mercado, a oferta de inovações por parte de diferentes redes se mostra um aspecto relevante. As inovações de produto/serviço por meio de redes dinamizam a oferta das empresas e permitem que haja maior vantagem competitiva no mercado (CHESBROUGH, 2010; GALLOUJ e DJELLAL, 2011; MILES, 2000).

As redes também possuem grande influência nas inovações de processo, porém há uma característica específica desse tipo de inovação: as redes mais influentes estão em contato com o cotidiano das empresas focais. $\mathrm{O}$ fato de a proximidade física da rede influenciar no impacto em inovações é uma das características dos dados coletados, a exemplo da influência das redes interpessoais informais e com clientes em comparação com redes com fornecedores e concorrentes. Além de tal característica, os dados coletados ilustram que inovações de processo são influenciadas por diferentes tipos de redes (BOEDDRICH, 2004; HOLMSTRÖM, 1998; PRÜGL e SCHREIER, 2006).

Já as inovações de marketing possuem uma abordagem diferente em relação às anteriores. Enquanto inovações de serviços são influenciadas pela maioria das redes e as de processo são influenciadas por redes que estão próximas do cotidiano das empresas 
focais, as inovações de marketing são influenciadas principalmente por redes organizacionais.

Essa característica, padrão nos dados coletados, é representada pela influência das redes com fornecedores, concorrentes e instituições nas inovações de marketing. Tal influência ocorre por peculiaridades de cada rede, a exemplo da divulgação de novos produtos apresentados pelos fornecedores, padrões de divulgação estabelecidos com os concorrentes e a cooperação das instituições na inovação das empresas.

O destaque das inovações de marketing é dado na baixa relevância das redes com clientes. Ao observar o comportamento contemporâneo dos consumidores em relação a marcas e empresas e a novas temáticas abordadas pela área de marketing, a influência exclusiva de redes organizacionais em inovações de marketing é um aspecto a ser aperfeiçoado nos entrevistados (SHETH, SISODIA, \& SHARMA, 2000; TIU WRIGHT, PIRES, STANTON e RITA, 2006).

As inovações organizacionais apresentaram os resultados mais extremos em comparação com os demais dados coletados. Enquanto as inovações anteriores possuem padrões de influência a partir dos diferentes tipos de redes, as inovações organizacionais são influenciadas exclusivamente por redes com instituições.

A partir das entrevistas realizadas, é possível identificar pouca abertura dos entrevistados em relação a influências internas e externas em questões organizacionais. Os entrevistados se mostraram protecionistas nesse aspecto, afirmando que são os únicos responsáveis pelas decisões organizacionais das empresas (DAMANPOUR, 1991).

Porém, são abertos a inserir inovações organizacionais a partir de redes com instituições, sendo representadas pelo projeto ALI do Sebrae-SP, interação com parques tecnológicos, universidades e demais cursos de desenvolvimento de negócios. Tais características ilustram que a amostra de pesquisa é pouco aberta em relação a diferentes estratégias de inovação, como a inovação aberta e a cocriação com concorrentes.

Além da Tabela 1, um mapa cognitivo causal foi criado para representação visual da interação entre variáveis. O mapa na Figura 1 representa apenas as interações entre variáveis que foram qualitativamente consideradas mais relevantes nas entrevistas realizadas. $\mathrm{Na}$ análise, os valores atribuídos para as interações foram desconsiderados, focando apenas nos depoimentos coletados durante as entrevistas. 
Figura 1 - Mapa Cognitivo Consolidado

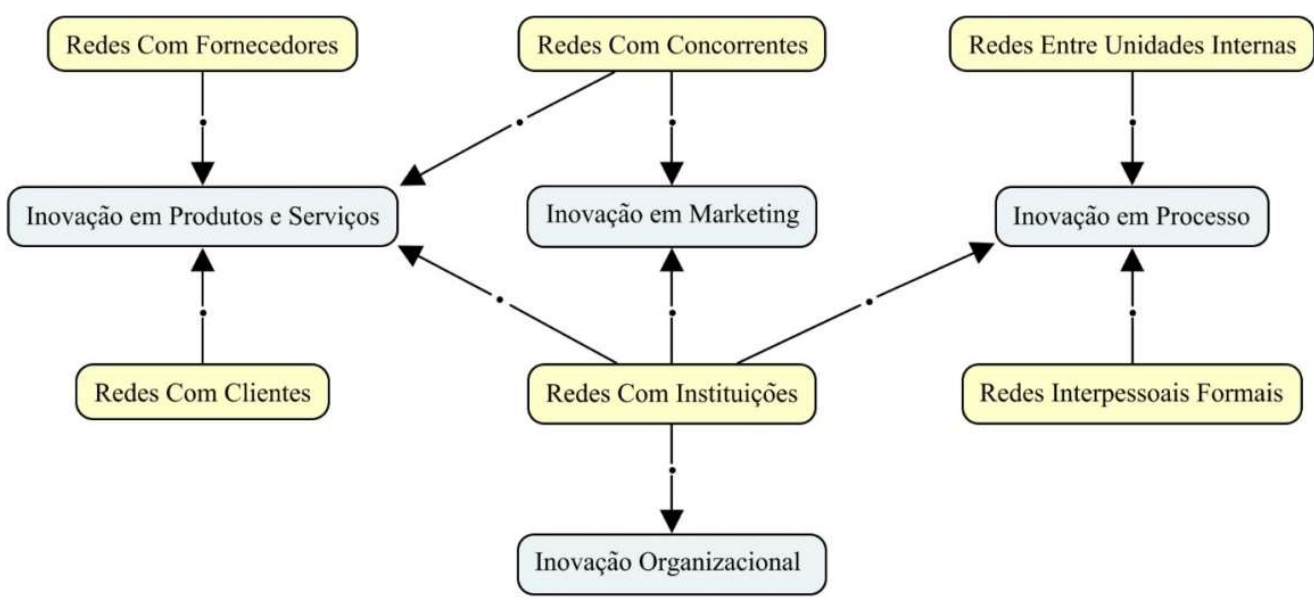

Elaborado pelos autores.

Apesar de a Tabela 1 apresentar os valores das interações entre variáveis, a Figura 3 possibilita a compreensão de como os entrevistados interpretam, de maneira prática, aspectos pontuais da contribuição das redes nos diferentes tipos de inovação.

O primeiro aspecto a ser considerado em relação à inovação de serviços é a influência exógena. Os entrevistados enfatizaram a relevância de redes com fornecedores, concorrentes e clientes para a implantação de novos serviços. O resultado corrobora a pesquisa de De Pellegrin et al. (2007) e Brass et al. (2004) sobre a influência de redes da cadeia produtiva na inovação, principalmente no que tange ao lançamento de novos produtos e serviços.

Em compensação, as inovações de processo são influenciadas principalmente endogenamente. As redes interpessoais formais e entre unidades internas são as mais valorizadas pelos entrevistados. Os processos, que tendem a ser técnicos e de menor compreensão por agentes externos, passam por inovações a partir de ideias internas ou para adaptação a inovações no serviço oferecido.

As inovações em marketing possuem duas influências principais: redes com concorrentes e instituições. O aspecto de maior destaque é a relevância de concorrentes nas empresas, principalmente no que tange à compreensão de novas estratégias de marketing e posicionamento de serviços no mercado. As redes com concorrentes reiteram a necessidade de compreensão das mudanças no ambiente estratégico descrita por Lemos da Silveira Santos et al. (2007). 
A inovação organizacional, por sua vez, é influenciada apenas por redes com instituições. A relevância de tais redes ilustra a necessidade de capacitação de empreendedores para todos os tipos de inovação e redução da mortalidade. A relevância de redes com instituições é potencializada pela baixa capacitação de empreendedores e concentração na inovação organizacional, conforme corrobora estudos de Pereira et al. (2009) e Filion (1999).

Com a Figura 1 é possível identificar, em aspectos gerenciais, quais os tipos de rede que são mais úteis para empreendedores que buscam aspectos específicos de inovação. Se uma MPE busca, por exemplo, inovar seus processos, as redes mais indicadas para serem construídas são entre unidades internas e interpessoais informais.

\section{CONSIDERAÇÕES FINAIS}

O estudo, cujo objetivo é analisar o impacto de redes interpessoais e interorganizacionais na inovação de MPEs brasileiras de serviços, alcançou resultados relevantes para a compreensão das peculiaridades e influência de redes na inovação, a partir de dados coletados com oito empreendedores de MPEs brasileiras de serviços.

Redes exógenas, cujos pares da empresa são agentes externos (como fornecedores, clientes e concorrentes), possuem maior influência na inovação de serviços porque estão relacionadas diretamente com o serviço a ser prestado pela empresa. Já redes endógenas, formadas por agentes internos (como as redes interpessoais formais e unidades internas da empresa), possuem maior influência na inovação de processos por conta da proximidade dos indivíduos com as tarefas cotidianas da empresa.

As redes com concorrentes, por sua vez, são mais influentes nas inovações de marketing, principalmente por conta da maximização da vantagem competitiva das empresas ao manter contato com seus concorrentes. Já as redes com instituições, representadas por parques tecnológicos, universidades e agências de apoio, são relevantes para todos os tipos de inovação por oferecerem a capacitação necessária para empreendedores as gerirem.

As áreas de redes e inovação são difundidas no Brasil, porém os conceitos aplicados a MPEs de serviços é a principal contribuição do estudo. Assim, é possível observar a manifestação das variáveis tanto para compreensão acadêmica das influências, investimentos gerenciais de empreendedores na área, como para o aperfeiçoamento de programas públicos de apoio a MPEs. 
Por conta de a amostra de pesquisa ser reduzida, apesar de contar com empresas de diferentes setores, não é possível generalizar os resultados para todas as MPEs brasileiras de serviços. Porém, caminhos para futuras pesquisas podem ser traçados em aspectos específicos, como: as diferenças de influência em MPEs de alta tecnologia (como a E3), as consequências da concentração decisória no empreendedor sobre inovação organizacional, ou a sustentabilidade do apoio institucional para a gestão de inovações.

\section{REFERÊNCIAS;}

Aranda, M. H. (2009). A importância da criatividade no processo de inovação (PI). Dissertação de Mestrado UFRGS.

Boeddrich, H. J. (2004). Ideas in the workplace: a new approach towards organizing the fuzzy front end of the innovation process. Creativity and innovation management, 13(4), 274-285.

Borgatti, S. P., Mehra, A., Brass, D. J., \& Labianca, G. (2009). Network analysis in the social sciences. science, 323(5916), 892-895.

Brass, D. J., Galaskiewicz, J., Greve, H. R., \& Tsai, W. (2004). Taking stock of networks and organizations: A multilevel perspective. Academy of management journal, 47(6), 795-817.

Camisón, C., \& Villar-López, A. (2014). Organizational innovation as an enabler of technological innovation capabilities and firm performance. Journal of Business Research, 67(1), 2891-2902.

Chesbrough, H. (2010). Open services innovation: Rethinking your business to grow and compete in a new era: John Wiley \& Sons.

Colossi, L. (2004). Características de ambientes organizacionais orientados ao comportamento criativo: o caso de uma empresa do setor de publicidade \& propaganda de Santa Catarina.

Cooper, D. R., \& Schindler, P. S. (2016). Métodos de Pesquisa em Administração-12 ${ }^{a}$ Edição: McGraw Hill Brasil.

Crescitelli, E., \& Figueiredo, J. (2010). Uso de diagramas causais na construção de um modelo de brand equity. Florianópolis/SC: EMA.

Damanpour, F. (1991). Organizational innovation: A meta-analysis of effects of determinants and moderators. Academy of management journal, 34(3), 555-590. 
De Medeiros, J. F., Ribeiro, J. L. D., \& Cortimiglia, M. N. (2014). Success factors for environmentally sustainable product innovation: a systematic literature review. Journal of Cleaner Production, 65, 76-86.

De Pellegrin, I., Balestro, M. V., Junior, J. A. V. A., \& Caulliraux, H. M. (2007). Redes de inovação: construção e gestão da cooperação pró-inovação. Revista de Administração, $42(3), 313-325$.

Eden, C. (1988). Cognitive mapping. European Journal of Operational Research, 36(1), $1-13$.

Emirbayer, M., \& Goodwin, J. (1994). Network analysis, culture, and the problem of agency. American journal of sociology, 99(6), 1411-1454.

Filion, L. J. (1999). Empreendedorismo: empreendedores e proprietários-gerentes de pequenos negócios. Revista de Administração, 34(2), 5-28.

Freeman, C. (1995). The 'National System of Innovation'in historical perspective. Cambridge Journal of economics, 19(1), 5-24.

Gallouj, F., \& Djellal, F. (2011). The handbook of innovation and services: a multidisciplinary perspective: Edward Elgar Publishing.

Gawer, A., \& Cusumano, M. A. (2014). Industry platforms and ecosystem innovation. Journal of Product Innovation Management, 31(3), 417-433.

Greenhalgh, T., Robert, G., Macfarlane, F., Bate, P., \& Kyriakidou, O. (2004). Diffusion of innovations in service organizations: systematic review and recommendations. Milbank Quarterly, 82(4), 581-629.

Gupta, S., Malhotra, N. K., Czinkota, M., \& Foroudi, P. (2016). Marketing innovation: A consequence of competitiveness. Journal of Business Research, 69(12), 5671-5681.

Holmström, J. (1998). Business process innovation in the supply chain-a case study of implementing vendor managed inventory. European journal of purchasing \& Supply Management, 4(2-3), 127-131.

Hoskisson, R. E., Hitt, M. A., Wan, W. P., \& Yiu, D. (1999). Theory and research in strategic management: Swings of a pendulum. Journal of management, 25(3), 417-456.

IBGE. (2015). Pesquisa de Inovação PINTEC - 2014. In I. B. d. G. e. Estatística (Ed.).

Kilduff, M., \& Tsai, W. (2003). Social networks and organizations: Sage.

Kotler, P., \& Keller, K. L. (2006). Administração de marketing.

Laperrière, A. (2008). Os critérios de cientificidade dos métodos qualitativos. POUPART, Jean et. al. A pesquisa qualitativa: enfoques epistemológicos e metodológicos, 2. 
Laukkanen, M. (2012). Comparative causal mapping and CMAP3 software in qualitative studies. Paper presented at the Forum Qualitative Sozialforschung/Forum: Qualitative Social Research.

Lemos da Silveira Santos, L., César Alves, R., \& Nunes Tavares de Almeida, K. (2007). Formação de estratégia nas micro e pequenas empresas: um estudo no centro-oeste mineiro. Revista de Administração de Empresas, 47(4), 1-15.

Lusch, R. F., \& Nambisan, S. (2015). Service Innovation: A Service-Dominant Logic Perspective. MIS quarterly, 39(1), 155-175.

Markoczy, L., \& Goldberg, J. (1995). A method for eliciting and comparing causal maps. Journal of management, 21(2), 305-333.

Markóczy, L. A. (1994). Barriers to shared belief: The role of strategic interest, managerial characteristics and organisational factors. The University of Cambridge.

Miles, I. (2000). Services innovation: coming of age in the knowledge-based economy. International Journal of Innovation Management, 4(04), 371-389.

OCDE. (2005). Manual de Oslo: Proposta de diretrizes para coleta e interpretação de dados sobre inovação tecnológica. OCDE. Organização para Cooperação $e$ Desenvolvimento Econômico: Departamento Estatístico da Comunidade Européia.

Pereira, M. F., Grapeggia, M., Emmendoerfer, M. L., \& Três, D. L. (2009). Fatores de inovação para a sobrevivência das micro e pequenas empresas no brasil DOI: $10.5585 /$ rai. v6i1. 245. RAI: revista de administração e inovação, 6(1), 50-65.

Porter, M. E. (1986). Estratégia competitiva: técnicas para análise de indústrias e da concorrência: Campus.

Provan, K. G., \& Kenis, P. (2008). Modes of network governance: Structure, management, and effectiveness. Journal of public administration research and theory, $18(2), 229-252$.

Prügl, R., \& Schreier, M. (2006). Learning from leading-edge customers at The Sims: opening up the innovation process using toolkits. $R \& D$ Management, 36(3), 237-250.

Rank, O. N. (2014). The effect of structural embeddedness on start-up survival: a case study in the German biotech industry. Journal of Small Business \& Entrepreneurship, $27(3), 275-299$.

Rattner, H. (1984). Inovação tecnológica e pequenas empresas: uma questão de sobrevivência. Revista de Administração de Empresas, 24(3), 70-73.

Rodan, S., \& Galunic, C. (2004). More than network structure: How knowledge heterogeneity influences managerial performance and innovativeness. Strategic management journal, 25(6), 541-562. 
Scavarda, A., Bouzdin-Chameeva, T., Goldstein, S., Hays, J., \& Hill, A. (2004). A review of the causal mapping practice and research literature. Paper presented at the Second world conference on POM and 15th annual POM conference.

Schumpeter, J. A. (1934). The theory of economic development: An inquiry into profits, capital, credit, interest, and the business cycle (Vol. 55): Transaction publishers.

Schumpeter, J. A. (1988). Capitalismo, sociedade e democracia. São Paulo: Abril Cultural.

SEBRAE. (2014). Participação das Micro e Pequenas Empresas na Economia Brasileira (pp. 1-108).

Sheth, J. N., Sisodia, R. S., \& Sharma, A. (2000). The antecedents and consequences of customer-centric marketing. Journal of the Academy of Marketing Science, 28(1), 55-66.

Tiu Wright, L., Pires, G. D., Stanton, J., \& Rita, P. (2006). The internet, consumer empowerment and marketing strategies. European Journal of Marketing, 40(9/10), 936949.

Tomaél, M. I., Alcará, A. R., \& Di Chiara, I. G. (2005). Das redes sociais à inovação. Ciência da informação, Brasília, 34(2), 93-104.

Un, C. A., \& Asakawa, K. (2015). Types of R\&D collaborations and process innovation: The benefit of collaborating upstream in the knowledge chain. Journal of Product Innovation Management, 32(1), 138-153.

Yin, R. K. (2013). Case study research: Design and methods: Sage publications. 